\title{
Weiteres über die Kinetik der Enzymwirkungen.
}

Von

S. G. Hedin.

(Der Redaktion zugegangen am 12. Dezember 1909.)

Bei monomolekularen katalytischen Reaktionen in einem homogenen Medium gelten folgende zwei Sätze:

1. Bei gegebener Katalysatormenge ist die Reaktionsgeschwindigkeit oder die in der Zeiteinheit umgesetzte Substratmenge der jeweiligen Substratkonzentration proportional.

2. Bei etwa durch Zugabe von Substrat konstant gehaltener Substratkonzentration ist die Reaktionsgeschwindigkeit der Konzentration des Katalysators proportional.

Bedeutet $\mathrm{C}$ die Substratkonzentration, $\mathrm{t}$ die Reaktionszeit und $\mathrm{k}$ eine von der Katalysatormenge abhängige Konstante, und ist $\mathrm{dG}$ die in der Zeit dt umgesetzte Substratmenge, so ist $-\frac{\mathrm{dC}}{\mathrm{dt}}$ die Reaktionsgeschwindigkeit. Nach dem Satz 1 ist also

$$
-\frac{\mathrm{dC}}{\mathrm{dt}}=\mathrm{k} \cdot \mathrm{C} \text {. }
$$

Wie ersichtlich, ist $-\frac{\mathrm{dC}}{\mathrm{dt}}$ oder die Reaktionsgeschwindigkeit $=\mathbf{k}$ für den Fall, daß $\mathrm{C}$ (durch Zugabe von Substrat) $=1$ gehalten wird, und $\mathrm{k}$ ist also unter dieser Voraussetzung die Reaktionsgeschwindigkeit. Nach dem zweiten der obigen Sätze ist also $\mathrm{k}$ der Katalysatormenge proportional. $\mathrm{k}$ wird der Geschwindigkeitskoeffizient genannt.

Der erste Satz ist auch für gewisse enzymatische Spaltungsprozesse bestätigt worden. Der Katalysator ist in diesem Falle das Enzym. Die Bedingung für die Gültigkeit des Satzes, daß die wirksame Enzymmenge während des ganzen Versuches dieselbe bleiben muß, trifft nur zu Anfang des Prozesses zu, 
und folglich ist auch die Gültigkeit der Regel zu dem Anfang begrenzt. Beispiele enzymatischer Prozesse, wo der erste Satz sich bewährt hat, sind: die Zerlegung von $\mathrm{H}_{2} \mathrm{O}_{2}$ durch Hämase ${ }^{1}$ ) und von, Glycylglycin durch Erepsin. ${ }^{2}$ )

Den zweiten der obigen Sätze habe ich auch für Enzymreaktionen als gültig erwiesen, nämlich für den Fall, daß bei gleichen Substratmengen die Zeiten gleichen Umsatzes den zugesetzten Enzymmengen umgekehrt proportional sich verhalten. ${ }^{3}$ ) Wie ich damals besonders hervorgehoben habe, ist die Herleitung streng genommen nur unter der Voraussetzung als richtig anzusehen, daß das eben angeführte Enzym-Zeitgesetz für verschiedene Stadien des Prozesses als zutreffend sich erweist. Dies bedeutet, daß der Prozeß mit verschiedenen Enzymmengen durch Nebenreaktionen und andere Störungen in der gleichen Weise beeinflußt wird und gewissermaßen dem gleichen Weg folgt; nur geht der Prozeß mit einer kleinen Enzymmenge langsamer als mit einer größeren. Daß dem so ist, habe ich für einige Fälle nachweisen können, nämlich für die Verdauung von Casein durch Trypsin in schwach alkalischer Lösung, für die Verdauung von in schwach alkalischer Lösung erhitztem und danach bis zu neutraler Reaktion dialysiertem Serumalbumin ebenso von in der gleichen Weise behandeltem Eierklar. $\left.{ }^{4}\right)$ Dagegen ist das Enzym-Zeitgesetz bei der Verdauung von Leim durch Trypsin nicht in dem gleichen Umfange gültig. ${ }^{5}$ )

\section{Weitere Versuche mit Trypsin.}

Im Anschluß an die eben erwähnten Versuche mit denaturiertem Serumalbumin habe ich einige weitere Untersuchungen mit dem gleichen Substrat ausgeführt. Das Albumin wurde in üblicher Weise durch fraktionierte Fällung mit $\mathrm{Am}_{8} \mathrm{SO}_{4}$ und Dialyse hergestellt. Dann wurde mit $0,1 \%$ igem $\mathrm{Na}_{2} \mathrm{CO}_{3}$

1) Senter, Zeitschrift f. physik. Chem., Bd. XLIV, S. 257, 1903.

2) Euler, Diese Zeitschrift, Bd. LI, S. 213, 1907.

3) Diese Zeitschrift, Bd. LVII, S. 468, 1908.

4) Journ. of Physiol., Bd. XXXII, S. 468, 1905.

5) Nicht publizierte Untersuchungen. 
$1 / 2$ Stunde auf dem Wasserbade erhitzt, um die hemmende Wirkung des Serumalbumins auf die tryptische Verdauung zu vernichten. Zur selben Zeit wird das Albumin durch Trypsin leichter verdaulich. Das Alkalicarbonat wurde so vollständig wie möglich wegdialysiert und, wenn nötig, neutrale Reaktion gegen Lackmus mit Säure hergestellt. Das Trypsin wurde durch Autolyse der Pankreasdrüse vom Ochsen und Dialyse bereitet, wobei genau darauf geachtet wurde, daß die Reaktion schließlich neutral sich erwies. Die Stickstoffmenge in $30 \mathrm{ccm}$ der klaren Trypsinlösung entsprach $1 \mathrm{ccm}$ 1/10-n-Säure. Bei den folgenden Analysen wurde der Stickstoff der angewandten Enzymmenge abgezogen.

Bereits bei meinen ersten Versuchen mit denaturiertem Serumalbumin fand ich, daß das Enzym-Zeitgesetz nicht zutrifft für den Fall, daß die Digestionsflüssigkeit alkalisch reagiert. Ist die Reaktion neutral, so entsteht früher oder später während der Verdauung ein Niederschlag, welcher allmählich unter dem Einfluß des Trypsins aufgelöst wird. Daß die Bildung des Niederschlages eine Folge der Trypsinwirkung ist, geht zur Genüge daraus hervor, daß derselbe bei Anwendung von gekochtem Trypsin ausbleibt. Außerdem steht die Zeit, nach welcher der Niederschlag zu entstehen beginnt, in einem gewissen Zusammenhang mit der Trypsinmenge. In vielen Versuchen habe ich die fragliche Zeit der Enzymmenge umgekehrt proportional gefunden. $\mathrm{Da}$ ich aber in anderen recht beträchtliche Abweichungen von dieser Regel gefunden habe, bin ich nicht geneigt, irgend welche Schlüsse aus diesen Versuchen zu ziehen, zumal das Einsetzen der Bildung des Niederschlages nicht eben leicht zu beobachten ist. Der Niederschlag ist in schwachem Alkali löslich und aus einer solchen Lösung durch Neutralisieren fällbar; ein Überschuß von Essigsäure löst den Niederschlag wieder. Bei neutraler Reaktion wird also der Niederschlag nur unter der Einwirkung des Trypsins weiter verändert. Diese Veränderungen des Substrates habe ich in der Weise verfolgt, daß ich nach Zeiten, welche den Enzymmengen umgekehrt proportional waren, das Digestionsgemisch filtriert und den Stickstoff in gleichen Volumina des wasser- 
klaren Filtrates bestimmt habe. Natürlich waren in allen zu vergleichenden Proben alle übrigen Bedingungen die gleichen; nur die Enzymmengen und die Digestionszeiten wurden variiert. Wie aus den folgenden Versuchen ersichtlich, waren die erhaltenen Stickstoffmengen nach der gleichen Zahl von EnzymZeiteinheiten dieselben; bedeutet $p$ die Enzymmenge und $t$ die Digestionszeit, ergaben folglich alle diejenigen Proben die gleichen Stickstoffmengen oder den gleichen Umsatz, für welche das Produkt $p \cdot t$ dieselbe Ziffer betrug.

\section{Versuch 1.}

Die Serumalbuminmenge war $25 \mathrm{ccm}$ und die Summe von Trypsin + zugesetztem Wasser betrug $40 \mathrm{ccm} .30 \mathrm{ccm}$ Filtrat wurde für die N-Bestimmung benutzt. Die Ziffern der letzten Kolumne geben die Stickstoffmengen in Kubikzentimetern 1/10-Normal-Säure an.

\begin{tabular}{c|c|c}
\hline $\begin{array}{c}\text { Enzymmenge } \\
\text { ccm }\end{array}$ & $\begin{array}{c}\text { Digestionszeit } \\
\text { Stunden }\end{array}$ & $\begin{array}{c}\text { Stickstoffmenge } \\
\text { ccm }\end{array}$ \\
\hline 30 & 24 & 34,45 \\
15 & 48 & 34,4 \\
10 & 72 & 34,35
\end{tabular}

$40 \mathrm{ccm}$ nicht filtrierter Digestionsflüssigkeit ergaben $39,3 \mathrm{ccm}$, welche Ziffer folglich der ganzen vorhandenen Stickstoffmenge entsprach. Die Zahlen der letzten Kolumne sind gleich, was beweist, daß der Niederschlag in allen Proben dieselbe Stickstoffmenge enthielt.

\section{Versuch 2.}

Die angewandten Volumina waren dieselben wie oben; nur wurden für die Stickstoffbestimmung $30 \mathrm{ccm}$ Filtrat genommen. (Siehe erste Tabelle S. 36.)

Die Stickstoffmenge in $30 \mathrm{ccm}$ nicht filtrierten Gemisches entsprach 38,8 ccm Säure und der Niederschlag enthielt demnach zu Anfang $(p \cdot t=5)$ mehr als die Hälfte der ganzen Stickstoffmenge. In diesem Versuch wurde auch die Stickstoffmenge des Gerbsäurefiltrates in Proben, welche der zweiten 


\begin{tabular}{|c|c|c|c|}
\hline & $\begin{array}{l}\text { Enzymmenge } \\
\text { ccm }\end{array}$ & $\begin{array}{l}\text { Digestionszeit } \\
\text { in Tagen }\end{array}$ & $\begin{array}{c}\text { N-Menge } \\
\text { ccm }\end{array}$ \\
\hline \multirow{3}{*}{$p \cdot t=5$} & 5 & 1 & 18,6 \\
\hline & 2,5 & 2 & 18,3 \\
\hline & 1,67 & 3 & 18,4 \\
\hline \multirow{3}{*}{$p \cdot t=10$} & 10 & 1 & 21,45 \\
\hline & 5 & 2 & 21,15 \\
\hline & 3,3 & 3 & 21,4 \\
\hline \multirow{3}{*}{$p \cdot t=15$} & 15 & 1 & 23,15 \\
\hline & 7,5 & 2 & 23,25 \\
\hline & 5 & 3 & 22,85 \\
\hline
\end{tabular}

Gruppe $(p \cdot t=10)$ gehörten, bestimmt. Die fraglichen Proben wurden also nach gehörigen Zeiten mit einem gewissen Volumen Gerbsäure gefällt, die Stickstoffmenge im gleichen Volumine der Filtrate bestimmt und die erhaltenen Ziffern für $30 \mathrm{ccm}$ der Digestionsflüssigkeit umgerechnet.

\begin{tabular}{c|c|c|c}
\hline \hline $\begin{array}{c}\mathrm{p} \\
\mathrm{ccm}\end{array}$ & $\begin{array}{c}\mathrm{t} \\
\text { (Tage) }\end{array}$ & $\begin{array}{c}\text { Obige Zahlen } \\
\mathrm{ccm}\end{array}$ & $\begin{array}{c}\text { Gerbsaures Filtrat } \\
\mathrm{ccm}\end{array}$ \\
\hline 10 & 1 & 21,45 & 14,45 \\
5 & 2 & 21,15 & 14,35 \\
3,33 & 3 & 21,40 & 14,45
\end{tabular}

Auch die Stickstoffmenge des Gerbsäurefiltrates zeigt also an, daß der Umsatz nach der gleichen Zahl von Trypsin-Zeiteinheiten der gleiche bleibt, was ich übrigens bereits vorher nachgewiesen habe. ${ }^{1}$ ) Die Stickstoffmenge des Gerbsäurefiltrates ist geringer als die des Filtrates von dem bei der Digestion gebildeten Niederschlag, weil die Gerbsäure gewisse von den wasserlöslichen Verdauungsprodukten ausfällt.

Mit Hilfe von zwei verschiedenen Analysemethoden ist es also zugleich bewiesen worden, daß die Veränderungen des Substrates nach dem Verlauf von der gleichen Zahl von Enzym-

2) Journ. of Physiol. (Engl.), Bd. XXXII, S. 473, 1905. 
Zeiteinheiten dieselben sind. Da außerdem beide Methoden für verschiedene Stadien der Reaktion diese Regelmäßigkeit ergeben, gilt also die Regel, daß der Geschwindigkeitskoeffizient der Enzymmenge proportional sich verhält nach der Herleitung, welche ich vorher gegeben habe. ${ }^{1}$ )

Die obigen Versuche wurden mit gekochtem und neutralisiertem Serumalbumin ausgeführt. Wenn man aber anstatt Serumalbumin eine Mischung von Serumalbumin und völlig neutralem (dialysiertem) Eierklar als Substrat verwendet, hat das Enzym-Zeitgesetz keine Gültigkeit mehr, wie folgender Versuch zeigt.

\section{Versuch 3.}

$25 \mathrm{ccm}$ gekochtes und neutralisiertes Serumalbumin wurde mit $5 \mathrm{ccm}$ Wasser resp. $5 \mathrm{ccm}$ neutralem Eierklar vermischt. $\mathrm{Zu}$ beiden Proben wurden $15 \mathrm{ccm}$ (Trypsin $+\mathrm{H}_{2} \mathrm{O}$ ) zugegeben, worauf unten angegebene Zeiten digeriert und mit $10 \mathrm{ccm}$ Gerbsäurelösung gefällt wurde. Dann wurde vom gebildeten Niederschlag filtriert und, wie oben, die N-Menge der Filtrate bestimmt.

\begin{tabular}{c|c|c|c}
\hline $\begin{array}{c}\text { Enzymmenge } \\
\mathrm{ccm}\end{array}$ & $\begin{array}{c}\text { Digestionszeit } \\
\text { in Tagen }\end{array}$ & $\begin{array}{c}\text { N-Menge } \\
\text { Serumalb. }+\mathrm{H}_{2} \mathrm{O} \\
\mathrm{ccm}\end{array}$ & $\begin{array}{c}\text { N-Menge } \\
\text { Serumalb. + Eierklar } \\
\mathrm{ccm}\end{array}$ \\
\hline 15 & 1 & 10,95 & 8,8 \\
7,5 & 2 & 10,8 & 5 \\
5 & 3 & 10,85 & 2,85
\end{tabular}

In allen Proben mit Serumalbumin + Eierklar finden wir also eine bedeutende Hemmung gegenüber den Proben ohne Eierklar, und zwar ist diese Hemmung am kräftigsten ausgesprochen in der Probe mit der geringsten Enzymmenge und sinkt mit zunehmender Menge. Dasselbe Verhältnis habe ich vorher gefunden bei Untersuchung der Hemmung, welche Eierklar auf die tryptische Verdauung des Caseins ausübt. ${ }^{2}$ )

$\mathrm{DaB}$ die Reaktionsgeschwindigkeit bei konstant gehaltener Substratkonzentration der Enzymmenge proportional sich ver-

1) Diese Zeitschrift, Bd. LVII, S. 468, 1908.

2) Diese Zeitschrift, Bd. LVII, S. 474, 1908. 
hält, bedeutet, daß die wirksame Enzymmenge der zugesetzten proportional ist. Dies ist also der Fall, wenn das Serumalbumin allein als Substrat vorhanden ist, aber nicht, wenn außerdem noch Eierklar zugesetzt wird. In der Gegenwart von Eierklar wird weniger Serumalbumin verdaut als ohne dasselbe und zwar ist der Unterschied größer, je kleinere Enzymmenge zugesetzt wurde. Nach der herrschenden Ansicht beruht die Hemmung auf einer Aufnahme des Enzyms seitens der hemmenden Substanz. In dem obigen Falle liegt also die Sache folgendermaßen. Die auf das Serumalbumin wirkende Enzymmenge ist, falls kein Eierklar zugegen ist, der zugesetzten Enzymmenge proportional. Ist Eierklar neben dem Serumalbumin vorhanden, nimmt dasselbe einen Teil des Enzyms an sich, und da das native Eierklar durch Trypsin praktisch unverdaulich ist, so folgt eine der mit dem Eierklar verbundenen Trypsinmenge entsprechende Hemmung der totalen Verdauung. Aus der stattgehabten Hemmung ist also zu erschließen, daß die durch das Eierklar aufgenommenen Enzymmengen prozentisch um so größer sind, je geringere Enzymmenge zugegeben wurde. Dasselbe gilt für den Fall, daß die Verdauung von Casein durch Eierklar gehemmt wird.

\section{Versuche mit Lab.}

Die Aufnahme von Enzym durch Substanzen, welche die Enzymwirkung hemmen, habe ich auch für das Lab untersucht und zwar, wie oben, mit Hilfe des Enzym-Zeitgesetzes. Für ein solches Studium ist das Lab insofern weniger geeignet als das Trypsin, als man mit dem Lab nur ein Stadium der Enzymwirkung, nämlich das im Gerinnungsaugenblicke für verschiedene Proben vergleichen kann, während beim Trypsin der ganze Verlauf des Umsatzes verglichen werden kann und tatsächlich unter gewissen Bedingungen gleich gefunden wurde. Beim Zusatz von hemmenden Substanzen finden beim Lab wie beim Trypsin Abweichungen vom Enzym-Zeitgesetz statt, welche auf andere Verteilung des Enzyms hindeuten. Als allgemeine Regel gilt auch hier, daß eine hemmende Substanz um so kräftiger wirkt, je geringere Enzymmenge vorhanden ist, oder daß der Hemmungs- 
körper von einer kleinen Enzymmenge prozentisch mehr an sich nimmt als von einer größeren. Diese Regel ist am deutlichsten ausgesprochen für den Fall, daß Hemmungskörper und Enzym vor dem Zugeben des Substrats (Milch) vermischt werden. Wird das Enzym erst nach dem Vermischen von Hemmungskörper und Milch zugesetzt, so kann es eintreffen, daß das Enzym-Zeitgesetz auch bei Gegenwart von Hemmungskörper gültig bleibt. Dies geschieht in solchen Fällen, wo die Enzymmenge im Vergleich mit dem Hemmungskörper groß ist, und es ist mir in den meisten Fällen gelungen, beim Gebrauch von größeren Mengen Hemmungskörper die oben angedeuteten $\mathrm{Ab}$ weichungen vom Enzym-Zeitgesetz nachzuweisen. Als Hemmungskörper dienten diejenigen Substanzen, welche ich bereits vorher als solche untersucht habe, nämlich Serum, Eierklar und Knochenkohle. ${ }^{1}$ ) Bereits wurden einige Versuche publiziert, welche die vorliegende Frage berühren, und aus diesen geht zur Genüge hervor, daß die eben genannten $A b w e i c h u n g e n$ vom EnzymZeitgesetz leicht nachweisbar sind für den Fall, daß das Enzym und Hemmungskörper zunächst vermischt werden. ${ }^{2}$ ) Folglich werden hier nur solche Versuche angeführt, bei welchen die Milch und der Hemmungskörper vor dem Zusatz des Labs vermischt wurden.

Im folgenden Versuche diente als Hemmungskörper P ferdes erum, das mit 40 Volumen Wasser verdünnt war. Bestimmungen der Gerinnungszeiten wurden ausgeführt einerseits mit Enzym + verdünntem Serum, anderseits mit Wasser anstatt Serum. Die letzteren Proben enthielten also

$10 \mathrm{ccm} \mathrm{Milch}+1 \mathrm{ccm} \mathrm{H}_{2} \mathrm{O}+1 \mathrm{ccm} \mathrm{Lab} \mathrm{.} \mathrm{.} \mathrm{A,}$ die ersteren

$10 \mathrm{ccm}$ Milch $+1 \mathrm{ccm}$ Serum $+1 \mathrm{ccm}$ Lab . . . B.

Die Konzentrationen der Lablösungen verhielten sich wie $1:{ }^{3 / 4}:{ }^{2 / 3}: 1 / 2: 1 / 3$. Die unter den Konzentrationen angegebenen Ziffern sind die Gerinnungszeiten; die nebenbei eingetragenen eingeklammerten Zahlen sind das Produkt Labmenge $X$ Gerinnungszeit. In der Serie A ist dieses Produkt konstant, was beweist, daß hier das Enzym-Zeitgesetz in Geltung ist; in der Serie B steigt

2) Diese Zeitschrift, Bd. LX, S. 85 u. 364, 1909.

2) Diese Zeitschrift, Bd. LX, S. 89, 365, 369. 
das Produkt mit fallender Enzymmenge, woraus folgt, daß die Gerinnungszeiten mit fallender Enzymmenge rascher steigen, als das Gesetz verlangt. Die Resultate waren:

Konzentration der Lablösungen.

\begin{tabular}{|c|c|c|c|c|c|}
\hline & 1 & $3 / 4$ & $2 / 3$ & $1 / 8$ & $1 / 8$ \\
\hline A & $\underset{11^{3 / 4}}{\operatorname{Min}}\left(11^{8 / 4}\right)$ & $\begin{array}{l}\text { Min. } \\
15^{1 / 4}\left(11^{7 / 16}\right)\end{array}$ & $\begin{array}{l}\text { Min. } \\
17^{1 / 2}\left(11^{2 / 3}\right)\end{array}$ & $\begin{array}{l}\text { Min. } \\
23^{1 / 2}\left(11^{3} / 4\right)\end{array}$ & $\begin{array}{c}\text { Min. } \\
35\end{array}$ \\
\hline B & $15 \quad(15)$ & $23 \quad\left(17^{1} / 4\right)$ & $27^{3} / 4\left(18^{1 / 2}\right)$ & $52^{1 / 2}\left(26^{1 / 4}\right)$ & $>180(>60)$ \\
\hline
\end{tabular}

Mit Eierklar des Hemmungskörpers wurde ein Versuch in der gleichen Weise wie mit Serum ausgeführt. Die Buchstaben $A$ und $B$ werden in derselben Bedeutung angewandt wie oben. Die gebrauchten Volumina waren:

$10 \mathrm{ccm} \mathrm{Milch}+1 \mathrm{ccm} \mathrm{H}_{2} \mathrm{O}+1 \mathrm{ccm} \mathrm{Lab} \mathrm{.} \mathrm{.} \mathrm{.} \mathrm{A}$ $10 »,+1 »$ Eierklar $+1 » » . .$. B.

Die Labkonzentrationen waren dieselben wie oben.

Konzentration der Lablösnngen.

\begin{tabular}{|c|c|c|c|c|c|}
\hline & 1 & $3 / 4$ & $2 / 3$ & $1 / 2$ & $1 / 3$ \\
\hline A & \begin{tabular}{|l|} 
Min: \\
$11^{3} / 4\left(11^{3} / 4\right)$
\end{tabular} & $\begin{array}{l}\text { Min. } \\
15^{1 / 2}\left(11^{7 / 16}\right)\end{array}$ & $\begin{array}{l}\operatorname{Min} \\
17^{1 / 2}\left(11^{2 / 8}\right)\end{array}$ & $\begin{array}{l}\text { Min. } \\
23^{1 / 2}\left(11^{8 / 4}\right)\end{array}$ & $\begin{array}{l}\text { Min. } \\
35\left(11^{2} / 8\right)\end{array}$ \\
\hline B & $13 \quad(13)$ & $18 \quad\left(13^{1 / 8}\right)$ & $22 \quad\left(14^{2} / 8\right)$ & $31^{1 / 4}\left(15^{5} / 8\right)$ & $60(20)$ \\
\hline
\end{tabular}

Mit Kohle habe ich kaum irgend welche Hemmung der Labwirkung für den Fall nachweisen können, daß die Kohle zunächst mit der Milch vermischt wird. Dies liegt daran, daß irgend welche Substanz in der Milch (Eiweiß?) durch die Kohle adsorbiert wird, was eine nachträgliche Aufnahme von Lab so gut wie vollständig verhindert, wie ich bereits vorher nachgewiesen habe. ${ }^{1}$ )

\section{Schlußfolgerung.}

Aus den angeführten Versuchen sowie aus anderen, die ich vorher publiziert habe, ist zu ersehen, daß das EnzymZeitgesetz, wenn Hemmungskörper zugegen sind, oft völlig versagt, und es wäre wohl möglich, daß die Nichtgültigkeit des Gesetzes in gewissen Fällen dem Vorhandensein von Hemmungskörper - im Substrat oder im Enzym - zuzuschreiben wäre.

1) Diese Zeitschrift, Bd. LXIII, S. 150, 1909. 